\title{
A Model for Value-Added E-Marketplace Provisioning: Case Study from Alibaba.com
}

\author{
Hong Hong Qing ${ }^{1}$ and Zi Shan Xue ${ }^{2}$ \\ ${ }^{1,2}$ College of Computer Science and Information Engineering, Chongqing Technology \\ \& Business University, Chongqing, P.R. China, 400067 \\ Qing.lily@163.com, xzs@ctbu.edu.cn
}

\begin{abstract}
Alibaba.com is one of the leading B2B e-Commerce companies in China. It provides an e-Marketplace connecting small and medium-sized buyers and suppliers both in China and around the world. Alibaba.com has grown admirably from its initial beginning as a general e-Market to a giant e-Marketplace. During its rapid growth, it has incurred challenges and became more exposed to greater competition. Currently the quality of its services and achieving profitability remain Alibaba's greatest challenges. In this paper we examine the key factors of the strategies of Alibaba.com, including trust, market, search, payment and tools. Alibaba's strategies led to its success: strong brand, providing customers with outstanding value and a superior shopping experience, massive sales volume and realizing economies of scale.
\end{abstract}

Key words: e-Marketplace, e-Commerce, Strategies.

\section{Introduction}

Alibaba.com, a member of the Alibaba Group of companies, is the world's leading B2B e-commerce company. Alibaba.com runs an international marketplace (www.alibaba. com) focusing on global importers and exporters, and a Chinese marketplace (www.alibaba.com.cn) focusing on suppliers and buyers trading domestically in China. Together, its marketplaces form a community of around 30 million registered users from over 240 countries and regions. In China, Alibaba.com runs a C2C and B2C marketplace (www.taobao.com), with over 62 million registered users.

Since launching in 1999 the marketplaces have built up a community of more than 24 million registered users and over 255,000 paying members by November 2007, Alibaba launched on the Hong Kong stock exchange and raised HK\$13.1 billion (US $\$ 1.7$ billion) in gross proceeds before offering expenses, making it the largest Internet IPO in Asia and the second largest globally.

Jack Ma, the founder of Alibaba, was first introduced to the Internet in 1995 when he went to Seattle as an interpreter for a trade delegation and a friend showed him the Internet. They searched for the key word "beer" on Yahoo and discovered that there were no data about China. He decided to launch a website and registered the name China Pages.

He borrowed \$2,000 to set up his first company and at the time knew nothing about personal computers or e-mails, and had never touched a keyboard before. He described the experience as "a blind man riding on the back of a blind tiger." 
Initially, the business did not fare well, since it was a part of China Telecom and Jack Ma reflects that: "everything we suggested, they turned us down, it was like an elephant and an ant."

He resigned, but in 1999, he gathered 18 people in his apartment and spoke to them for two hours about his vision. Everyone put their money on the table, and he got $\$ 60,000$ to start Alibaba.

He chose Alibaba as the name since the name was easy to spell and associated with "Open, Sesame," the command that Ali Baba used to open doors to hidden treasures in One Thousand and One Nights.

During the dot-com bubble, there were layoffs, such that by 2002 there was only enough cash to survive for 18 months. Alibaba had a lot of free members using alibaba.com, and Alibaba didn't know how they would make money. But they then developed a product for China exporters to meet U.S. buyers online, which saved the company. Alibaba made only $\$ 1$ in profits in 2002. By the end of 2004, Alibaba made $\$ 1$ million a day profit. Each year since it has improved in profitability to the position where it was launched on the stock market.

Today, Jack Ma's vision is to build an e-commerce ecosystem that allows consumers and businesses to do all aspects of business online. They are partnering with Yahoo and have launched online auction and payment businesses. His vision is expansive, he says: "I want to create one million jobs, change China's social and economic environment, and make it the largest Internet market in the world" [1].

Building a model for value-added e-Marketplace provision is one of the key elements of successful e-Marketplace. The speed and strategy of the customer frontline change have placed the focus on the process and a possible need for a closer integration between e-Marketplace and customer-close activities.

The present study considers the strategies of the model of Alibaba's e-Marketplace, focusing on the e-Marketplace development processes and effective operation. The target groups are the companies within the Alibaba e-Marketplace, mainly SMEs. Most SMEs use new technology and multiple channels extensively to do business with customers, and are in general, Alibaba.com with new business models. Thus they can provide an interesting comparison with other models.

The purpose of the present study is to find Alibaba.com success factors, best-practice example and areas of improvement for the coordination of the business and e-Marketplace development processes, by investigating:

1. The development process, with a focus on the strategic stages of e-Marketplace.

2. Problems with, and solutions for, across product lines and services,

3. Problems, solutions and co-ordination of purchaser-supplier relations, and

4. The possible effects of customer front-line changes with reference to the co-ordination of processes between the business and management

This paper is only an initial presentation of the results, with analysis and categorization of key success factors.

\section{Overview of the Online Marketplace}

The population of Internet and World Wide Web users has risen fast since 1990s with the development and advancement of computer technologies. Many firms have 
launched their businesses through the Internet because of these technological innovations. This resulted in a wide variety of product offerings from Internet Businesses online. Online purchasing has grown at an amazing rate. Therefore, companies that carry out online business have great opportunities to achieve success. Conversely, there are great competition and threats as well when a company chooses to enter online business [2].

E-Business is one of the flourishing industries. Barriers to entry into this industry are quite high, and suppliers have modest influence over suppliers. The introduction of the Internet has brought about many changes to this industry because it has increased rivalry, purchasing capability of buyers and substitutes.

The Chinese suppliers offer the largest products market. Chinese suppliers on the Internet are fragmented; most of Chinese suppliers conduct their business through traditional exporters. With the development of the Internet, buyers and sellers now have a quick and timely communication channel, and switch to conduct business online. Thus, more and more business companies favor to do business on an e-Marketplace.

From the mid-1990s, The Chinese government has advocated formation of industry focused websites. There are some websites that offer B2B for import and export companies. e.g. http://www.iexportcn.com/, http://www.gjtrade.net/ etc. Since these e-Marketplaces can reduce intermediates like wholesalers, business companies can sell their products at a discounted price. Because of this strategy, the volume of B2B transactions reached RMB $¥ 1.25$ trillion in 2007, a growth rate increase of $25.5 \%$ from 2006. In addition, according to their self-report, they are growing almost 25 to 35 percent a month. Many independent stores have gone out of business because of this trend. Such scenarios set the stage for the success of Alibaba.com [3]. The real opportunity created by Alibaba.com is its own unique Web site management methods. Jack Ma led his team to implement their e-Marketing strategies to meet the needs of a group of SMEs, and they have got great benefits from its e-Marketplace [4].

It is necessary to understand the e-Marketplace industry as a whole and the competitors in the e-Marketplace as well in order to remain competitive in the market. One of Alibaba.com's largest competitors is vertical e-Marketplaces, i.e. industry enterprise website, which obviates the fact that Alibaba should build an e-Commerce ecosystem that allows consumers and businesses to do all aspects of business online.

\section{Research Method}

The present study is based on the study of a series of reports from Alibaba. The paper aims at understanding the experience of using alibaba.com processes and their strategies. A qualitative research method has been employed in this study.

In each report, personal interviews were carried out with persons from the SMEs as well as those from business organizations, which helps to enable exploration and comparison of the internal processes and capabilities from two different viewpoints, including the customer view (e-Marketing) and the management view (e-Marketplace). The analysis is based on the results which are obtained from interviews with SMEs and Alibaba.com. 


\section{Alibaba.com Strategies Analysis}

E-Business has been expanding at a phenomenal rate during 2000's. New on-line business were opening and growing into firms on e-Marketplaces previously not served by any website. The number of new products each year grew because new suppliers increasingly found their way into export and import markets. Similarly, the profit for sellers rose by robust percentages, and B2B industry began evolving into the maturity stage of the industry life cycle in the mid of 2000s. Jack Ma, CEO of Alibaba Group, predicts that Internet product purchases will grow exponentially over the next few years, and he proposes the strategic framework to enhance the competitiveness of Alibaba (figure1).

Alibaba's five forces of strategy framework is a valuable way in analyzing the industry, and the five forces are competition from trust, market, search, payment and tools.

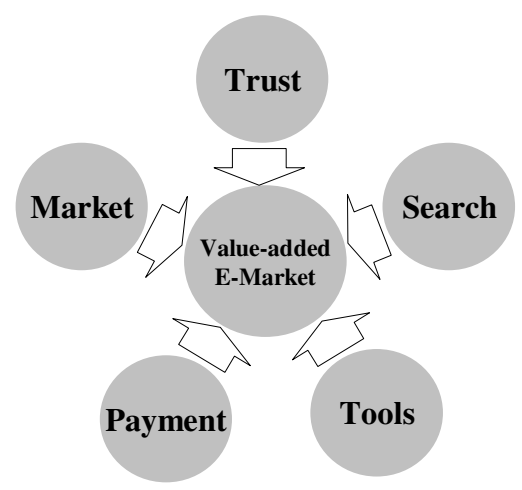

Fig. 1. Alibaba.com Company Strategies

\subsection{Trust}

For E-Marketplaces to win customers credibility is required, rather than purely financial or technological benefits. Due to the lack of Internet laws, web-based transactions have to face a serious credibility crisis. Alibaba announced the establishment of an online credit integrity system for the business community in 2002 ; it is a credibility-scheme, based on confidence-building mechanisms. It is called "China TrustPass membership".

Similar to the corporate version, entrepreneurs need to pass a third-party authentication and verification process before they become a China TrustPass member. As part of this process applicants need to provide their identity card and bank information. Once they become an authorized China TrustPass member, Alibaba.com will maintain an online trust rating for individuals to build confidence and credibility with potential buyers.

Alibaba.com launched its original China TrustPass membership in 2002 and as of March 31, 2008 had more than 280,000 paying members. Alibaba.com's China marketplace has close to 25 million registered users and is growing at over $36 \%$ year-on-year. The price of the new service is RMB 2,300 per year, slightly lower than the corporate version which currently sells at RMB 2,800. 
June 10, 2008, Alibaba.com announced the launch of a new service called "China TrustPass for Individuals" on its Chinese-language online marketplace, which connects suppliers and buyers trading domestically in China. Until now, Alibaba.com's China TrustPass membership had only been available to corporate users [5] [6].

\subsection{Market}

Avoiding the most popular portal model, Alibaba adhered to B2B and C2C for SMEs and help them to quickly find business opportunities in a global market. Three websites were launched from 1999 to 2003: Global import and export enterprises and enterprises in the e-Marketplace (www.alibaba.com, 1999), Domestic trade enterprises and enterprises in the e-Marketplace (www.alibaba.com.cn, 2000) Terminal between consumers and retailers of the e-Marketplace (www.taobao.com, 2003). SMEs have gained huge profits from Alibaba e-Marketplaces.

As of June 30, 2008, Alibaba.com's international marketplace had 29,766 Gold Supplier members, a net increase of 7,748 from 22,018 as of June 30, 2007. The number of International TrustPass members reached 13,912 as of June 30, 2008, a net increase of 2,953 from 10,959 as of June 30, 2007.

Alibaba.com's China marketplace had 324,328 China TrustPass members as of June 30, 2008, a net increase of 101,752 from 222,576 as of June 30, 2007. The strong growth in China TrustPass members is due to both external and internal factors. Externally, the increased Internet penetration in China and a continued strong domestic economy is benefiting Alibaba.com's business. Internally, it is seen that improvements in customer acquisitions through Alibaba's sales force and their network of resellers. The sales of value-added services such as keyword bidding and premium placements also recorded healthy growth. In addition, the launch of China TrustPass for Individuals' in the second quarter added over 10,000 paying members as of June 30, 2008 [7][8].

\subsection{Search}

August 2005, a strategic partnership between Alibaba and China Yahoo, Alibaba has therefore become China's largest Internet company. Similarly, Yahoo Alibaba in the future of industrial layout also has important significance. While most people have never viewed eBay as a search engine, but in fact it approximately deal with 130 million searches every day from all over the world, equivalent to Google's search volume. Many of the businessmen who sell on eBay, every year on Google bid for ads to fight for PPC. At present, Alibaba have made a series of adjustments to yahoo.com.cn with powerful search technology, E-mail, IM tools, and PPC advertising business, though its future business model has not yet been determined, but no doubt it will be the practice of the theory of Jack Ma "e-Commerce search is the electronic search business".

\subsection{Payment}

A lack of payment confidence is a sensitive issue. Alibaba.com created the third-party payment platform (www.alipay.com). It is responsible for the funds between parties to the transaction. October 2003, Alipay.com partnered with the Industrial and Commercial Bank of China, China Construction Bank, Agricultural Bank and China Merchants Bank, 
China Construction Bank, the International Organization of VISA International. This means that since the International Organization of VISA International, and other major financial institutions established a strategic partnership with Alipay.com, it has become the country's largest independent third-party electronic payment platform. As of December 2005, the daily average transaction volume through Alipay.com has been more than $¥ 20$ million, and still growing rapidly.

\subsection{Tools}

January 2007, Alibaba.com established Alisoft.com in order to improve the levels of SMEs management. Alisoft.com offer new SaaS (software as a service) model for the majority of SMEs to provide full life cycle of software services, and at the same time meet their e-Commerce and enterprise management needs.

August 2, 2008, Alibaba.com announced the beta launch of Export-to-China, a new service which will enable international entrepreneurs and small and medium-size enterprises (SMEs) to sell direct to China's growing number of buyers [9].

\section{Alibaba.com Industry Analysis and Discussion}

Alibaba.com identifies the key success factors in its business model such as a strong brand, providing customers with outstanding value and a superior shopping experience, massive sales volume and realizing economies of scale.

Alibaba.com is the only e-Marketplace which includes B2B, B2C, and C2C. Alibaba is the world's No.1 international trade and domestic trade e-Marketplace in China according to Forbes Statistics. Additionally, according to Chinese Academy of Social Sciences "2005 Report on the e-Commerce Analyst Report", Alibaba.com is estimated to have over 72 percent of the $\mathrm{C} 2 \mathrm{C}$ market and 50 percent of the $\mathrm{B} 2 \mathrm{~B}$ market in China [10].

However, Jack Ma's vision, has evolved much further than just being the world's largest e-Marketplace. The vision is to be the world's largest single e-Marketplace for online shoppers and to become a premier all-purpose e-Business by leveraging its existing brand and business model.

Alibaba.com has several core values which play a crucial part in the success of the company. The first one is excellent customer service based on extraordinary technology. The company provides various services such as product search and safe payment. In addition, the company also provided business information containing descriptions, snippets of reviews and testimonials posted by sellers and buyers.

Convenience and price is the second core value of the company. It manages the e-Marketplace over the Internet and has its own distribution system. Similarly, Alibaba does not have to spend too much money on real estate and other operation costs because it is pursuing Internet-based business. Thus, it can reduce SMEs' inventory expenses.

On one hand, the company has several key resources and capabilities to meet the challenges presented by opportunities and threats. These resources and capabilities have thus far allowed the company to be the world leader in the special e-Marketplace of B2B and C2C industry. Jack Ma, founder and chairman of Alibaba since 1999, is the driving force behind the company. He is a valuable resource that gives the company the competitive advantage with above-average return. 
Alibaba.com has also a very strong brand name presence in the e-Marketplace of B2B, B2C and C2C which is primarily due to their successful exploitation of their "first-mover" advantage. It would be able to leverage this brand name as it realizes its plans for expansion in the future.

In addition, the technological infrastructure of Alibaba also gives its competitive advantage against the other rivals. It can open a new e-Marketplace with different products offerings very easily because its core search technologies are easily re-usable.

Its website is elegantly designed, easy to navigate and quick to load. It also has numerous proprietary inventions like the click shopping, personalized recommendations and user rating which make shopping more pleasurable [11].

Alibaba's strategies, such as trust, market, search, payment and tools, strategic partnership, compelling value, and active advertising implemented by the company have contributed to its success in sales growth and cost efficiency.

However, transitioning the company culture is one of the problems and weaknesses encountered by Alibaba.com. A lot of changes have to be made in order for Alibaba to adjust to different situations especially since the rules of business keep changing rapidly. Alibaba was only a start-up few years ago but it grew rapidly and successfully in the e-Business. Thus, Alibaba should no longer be considered a start-up but a company moving from one culture to another because of its expansion and time in the industry [12].

Another problem and weakness is the financial difficulties brought about by its international exposure. The fluctuation of exchange rates from different currencies into RMB may affect Alibaba's money market. The success of Alibaba's international expansion also depends on local economic and political conditions.

\section{Conclusion}

Alibaba.com has grown admirably from its initial beginnings as a small e-Marketplace into a giant e-Marketplace. During this process of rapid growth, it has becomes more exposed to greater competition. China's role in the global market is evolving from a top manufacturing hub and exporter into a powerful global buyer as well. China's growing demand for imported goods is being fueled by a booming economy, an emerging middle class, the rising Chinese Yuan and favorable government measures promoting imports. Cutting costs and achieving profitability remain Alibaba's greatest challenges. However, there are key factors such as a strong brand, providing customers with value-added e-Marketplace, outstanding value and a superior shopping experience, massive sales volume and realizing economies of scale that all contributes a lot to the success of this company. These factors and the people around the company enable Alibaba.com to face the threats presented by competitor e-Marketplaces. Essentially, the company should aim to maintain its gross margins in its existing business and in future product lines such as the TrustPass system, the AliPay system, powerful search technology, E-mail, IM tools, and PPC advertising etc. In order to do this, Alibaba.com should develop strategic partnerships with all of its main suppliers and its customers to build an e-commerce ecosystem that allows consumers and businesses to do all aspects of business online. 


\section{References}

1. Chaffey, D.: Alibaba case study - E-commerce marketplace in China, http: / / www. davechaffey.com/

2. Zeng, M.: The Future Internet will be e-Commerce (February 29, 2008), http://aliresearch.com/

3. The E-Businessmen Conference Organizing Committee, 2008 Report on the Development of e-Market in China, pp. 1-18 (July 2008)

4. Fang, D.X.: The Arrival of Third Wave of China's Internet, Peking University Business Review. Peking University Press (April 25, 2008), http: / / aliresearch. com/

5. Alibaba.com Launches New China TrustPass Product for Individuals (June 10, 2008), http: / / www.ir.alibaba.com/

6. Xiao, Y.: Alibaba.com Announces 2008 Interim Results (July 16, 2008), http: / / www.aliresearch.com

7. Liang, C.X., Song, F.: Businessmen on the Internet will Win the World, June 2008, pp. 43-59. China Citic Press (2008)

8. Alibaba.com Announces 2008 Interim Results (August 27, 2008), http: / /ir.alibaba.com/

9. Alibaba.com Helps Small and Medium Businesses Sell Direct To China (August 2, 2008), http: //ir.alibaba.com/

10. China's Social Science Research Institute, 2005 Report on the e-Commerce, 184-265 (December 2005)

11. Liang, C.X., Song, F.: Businessmen on the Internet will Win the World, June 2008, pp. 15-23. China Citic Press (2008)

12. Jing, L.B.: Alibaba further expansion of the value chain (June 6, 2008), http: //aliresearch.com 